\title{
Cloud Model-Based Energy Management Strategy for Parallel Hybrid Vehicles
}

\author{
Xiaolan Wu, ${ }^{1}$ Guifang Guo, ${ }^{2}$ and Zhifeng Bai' \\ ${ }^{1}$ School of Mechanical and Electrical Engineering, Xian University of Architecture and Technology, Xian, Shaanxi 710055, China \\ ${ }^{2}$ School of Information Engineering, Tibet University for Nationalities, Xian, Shaanxi 710055, China \\ Correspondence should be addressed to Xiaolan Wu; wuxl2011@126.com
}

Received 22 July 2014; Accepted 20 November 2014

Academic Editor: Onur Toker

Copyright (c) 2015 Xiaolan Wu et al. This is an open access article distributed under the Creative Commons Attribution License, which permits unrestricted use, distribution, and reproduction in any medium, provided the original work is properly cited.

\begin{abstract}
Using the uncertain conversion capacity between the expressions of quantitative and qualitative concept in the cloud model, an energy management strategy based on cloud model is developed for parallel hybrid vehicles (PHVs). By the driver input and the state of charge (SOC) of the energy storage, a set of rules are developed to effectively determine the torque split between the internal combustion engine (ICE) and the electric motor. An analysis of the simulation results is conducted using ADVISOR in order to verify the effectiveness of the proposed control strategy. It is confirmed that the control scheme can be used to improve fuel economy and emission of the hybrid vehicles.
\end{abstract}

\section{Introduction}

Growing concerns on environmental issues and energy crises have forced more and more researches to focus on new generation vehicles such as electric vehicle (EV), hybrid electrical vehicle (HEV), and fuel cell electric vehicle (FCEV). Since EV has the shortcoming of short range due to the handicap of battery technology and FCEV is in its early development stages, the HEV seems to be the viable alternative to the ICE automobile at present because of its potential to reduce fuel consumption and environmental pollution.

To fully realize the potential of HEVs, the design of energy management strategy (EMS) is very crucial. The EMS determines the energy flow between all components in order to fulfill the power balance between the load power and the power source. Many research efforts into the EMSs of parallel hybrid vehicle (PHV) have been conducted in recent years. They can be roughly classified into three categories. The first type is rule-based methods [1]. The second type employs intelligent control technology (fuzzy logic, neural networks, agent, etc.) for power distribution of PHVs [2-5]. The third approach is based on optimization methods such as genetic algorithm and dynamic programming $[6,7]$. The fourth approach is based on modern control theory such as optimal control, decoupling control, and robust control [8$10]$.

Cloud theory, first formulated by De-yi et al. based on the membership function of fuzzy set theory [11], is a model of the uncertainty transformation between quantitative representation and qualitative concept using language value. It is successfully used in many fields, such as intelligence control $[12,13]$, data mining $[14,15]$, target recognition $[16]$, pervasive computing [17], and intelligent algorithm improvement [18, 19].

In this paper, a novel EMS based on cloud model is proposed for hybrid electric vehicle. This paper is organized as follows. Section 2 describes the configuration of PHV. Section 3 presents cloud theory. The proposed EMS is given in Section 4. Simulation results and analysis are shown in Section 5. Section 6 is the conclusions.

\section{Parallel Hybrid Vehicle Configuration}

Figure 1 presents the configuration of a PHV with an electric motor (EM), an internal combustion engine (ICE), and transmission. In this case, both EM and ICE may deliver power to the vehicle wheels. The EM may also be used as 


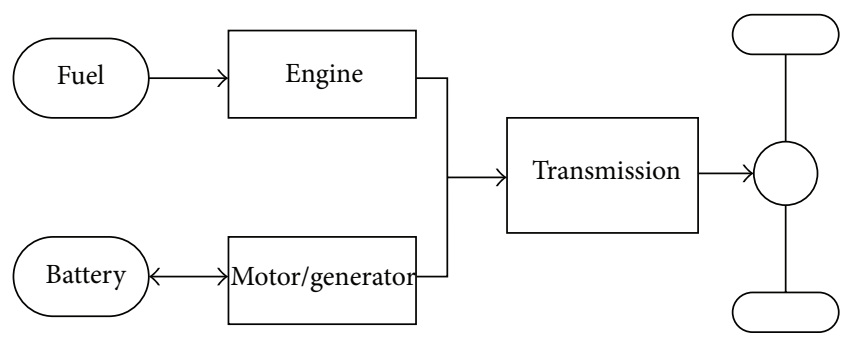

Figure 1: Parallel HEV configuration.

a generator to charge the battery by either the regenerative braking or absorbing the excess power from the engine when its output is greater than that required to drive the wheels. The main advantage of PHV is improved dynamic performance due to the direct coupling between the ICE, EM, and the wheels.

The following parameters of main components are used for this study:

(i) body mass $592 \mathrm{~kg}$;

(ii) rolling resistance coefficient 0.009 ;

(iii) body aerodynamic drag coefficient 0.335 ;

(iv) vehicle front area $2 \mathrm{~m}^{2}$;

(v) wheel radius $0.282 \mathrm{~m}$;

(vi) five speed manual gearboxes $2.84,3.77,5.01,7.57$, and 13.45

(vii) SI engine $36 \mathrm{~kW}$;

(viii) AC motor $17 \mathrm{~kW}$ peak;

(ix) Hawker Genesis VRLA battery 16 12V26Ah10EP.

The size of the engine, electric motor, and the number of battery modules are determined based on a previous study through an optimization approach [20].

\section{Cloud Theory}

Cloud is a model that expresses the uncertainty transition between qualitative concept and quantitative representation by using natural language. A piece of cloud is made up of many cloud drops that represent a realization of a qualitative concept. The realization has uncertain factors, that is, fuzziness and randomness [12].

3.1. Digital Characteristics of the Membership Clouds. Let $U$ be the set, $U=\{u\}$, as the universe of discourse, and $T$ a linguistic term associated with $U$. The mapping $C_{T}(x): u \rightarrow$ $[0,1]$, for all $x \in C_{T}(x)$, namely, the random distribution of $C_{T}(x)$ in $U$, is called membership cloud, briefly called cloud. When $C_{T}(x)$ obeys the normal distribution, cloud is called normal cloud model. It is expressed with three digital characteristics, expected value $E_{x}$, entropy $E_{n}$, and hyperentropy $H_{e}$ [11].

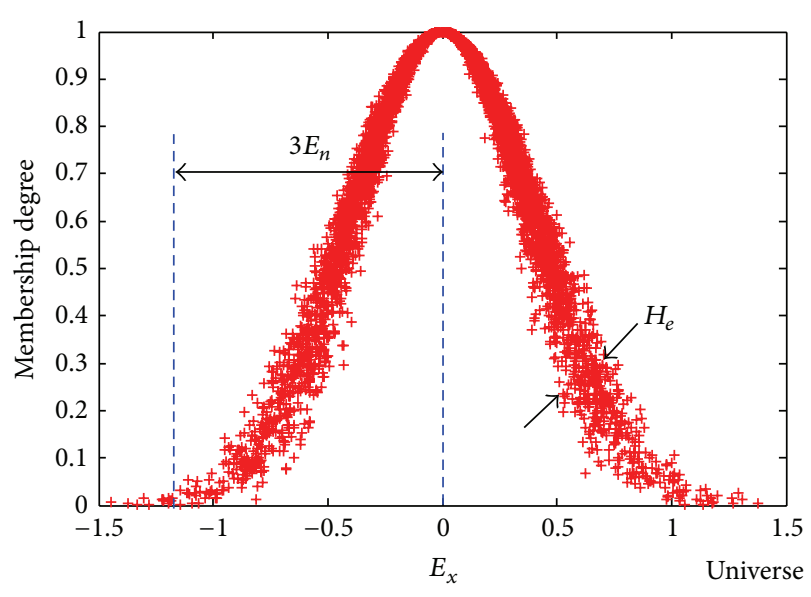

FIGURE 2: Three digital characteristics of normal cloud.

Suppose $R_{1}\left(E_{x}, E_{n}\right)$ express the random function obeying normal distribution; then

$$
\begin{gathered}
x_{i}=R_{1}\left(E_{x}, E_{n}\right), \\
P_{i}=R_{1}\left(E_{n}, H_{e}\right), \\
\mu_{i}=e^{-(1 / 2)\left(\left(x_{i}-E_{x}\right) / P_{i}\right)^{2}} .
\end{gathered}
$$

Cloud model composed of $\operatorname{drop}\left(x_{i}, \mu_{i}\right)(i=1,2, \ldots)$ is called one-dimension cloud model, denoted by $\left(E_{x}, E_{n}, H_{e}\right)$ [21]. The typical example of one-dimension normal cloud model and its digital characteristics $\left(E_{x}=0, E_{n}=0.4\right.$, and $\left.H_{e}=0.04\right)$ are shown in Figure 2. The expected value $E_{x}$ is the position at $U$ corresponding to the center of gravity of the cloud. The entropy $E_{n}$ is a measure of the coverage of the concept within the universe of discourse. The hyperentropy $H_{e}$ is the entropy of $E_{n}$, which is a measure of dispersion of the cloud drops.

Suppose $R_{2}$ express two-dimension random function obeying normal distribution; then

$$
\begin{gathered}
\left(x_{i}, y_{i}\right)=R_{2}\left(E_{x}, E_{y}, E_{n_{x}}, E_{n_{y}}\right), \\
\left(P_{x_{i}}, P_{y_{i}}\right)=R_{2}\left(E_{n_{x}}, E_{n_{y}}, H_{e_{x}}, H_{e_{y}}\right), \\
\mu_{i}=e^{-(1 / 2)\left[\left(x_{i}-E_{x}\right)^{2} / P_{x_{i}}^{2}+\left(y_{i}-E_{y}\right)^{2} / P_{y_{i}}^{2}\right]} .
\end{gathered}
$$

Cloud model composed of $\operatorname{drop}\left(x_{i}, y_{i}, \mu_{i}\right)(i=1,2, \ldots)$ is called two-dimension cloud model, where $E_{x}$ and $E_{n_{x}}$ are expected value, $E_{n_{y}}$ and $E_{y}$ are entropy, and $\left(E_{x}, E_{n_{x}}, H_{e_{x}}\right)$ and $\left(E_{y}, E_{n_{y}}, H_{e_{y}}\right)$ are two relatively independent onedimensional cloud.

3.2. Cloud Generator. Cloud generator includes positive and backward cloud generator, which is used for realizing the transform between quantitative value and qualitative concept. As shown in Figures 3 and 4, given three characteristics $E_{x}, E_{n}$, and $H_{e}$, the positive cloud generator can produce the required cloud drops; accordingly, the backward cloud 


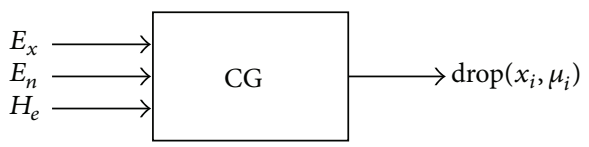

FIgURE 3: The positive cloud generator.

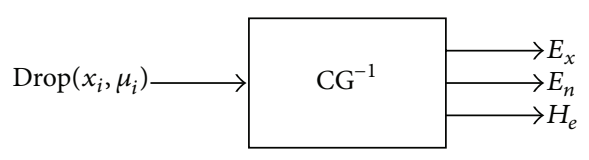

FIGURE 4: The backward cloud generator.

generator can get digital characteristics from the given cloud drops. The positive cloud generator also contains two kinds of generator, $X$-condition generator when given the numerical value $x$ in the universe of discourse and $Y$-condition generator if the membership degree $\mu$ is given [14].

The above-mentioned cloud generator is for onedimension case. As for two-dimension case, its positive cloud is produced by two group characteristics $\left(E_{x}, E_{n_{x}}, H_{e_{x}}\right)$ and $\left(E_{y}, E_{n_{y}}, H_{e_{y}}\right)$; the backward cloud generator produces two group data. $X$-condition cloud needs to be given $x$ and $y$ value; $Y$-condition cloud gets drops by being given one or two membership degree values.

3.3. Rule Constructor of Cloud Model. The rule constructor of cloud model is composed of $X$-condition and $Y$-condition cloud generator. It can be divided into one-dimension, twodimension, and multidimension according to the different combination. For example, one-dimension single rule is implemented by connecting a one-dimension $X$-condition cloud generator and a one-dimension $Y$-condition cloud generator, when a two-dimension $X$-condition cloud generator combining a one-dimension $Y$-condition cloud generator is called two-dimension single rule constructor. The multirule constructor of cloud model is constituted by two or more single rule constructors, which can reflect multicomplicated rules as follows:

$$
\begin{aligned}
& \text { IF } A_{1} \text { and } B_{1} \text { THEN } C_{1} \text {, } \\
& \text { IF } A_{2} \text { and } B_{2} \text { THEN } C_{2} \text {, }
\end{aligned}
$$

where $A_{i}$ and $B_{i}$ are linguistic concepts represented by cloud models. Figure 5 shows a two-dimension multirule constructor. Using these multirule constructors, intelligence control based on cloud model can be implemented [22].

\section{Cloud Model-Based Energy Management Strategy}

This section describes the EMS based on cloud theory for a parallel hybrid vehicle. The energy in the system should be managed in such a way that the power demand from the driver is satisfied consistently, the battery SOC is maintained within desired operating range, and the overall system efficiency is optimal.

4.1. Description of Power Controller. The power controller in this study is used to determine the proper torque split to cause the ICE to work possibly in the vicinity of its optimal operating points at all times. The optimal operating points are determined based on ICE parameters at the current vehicle speed, so as to minimize instantaneous fuel consumption and emissions [23]. Figure 6 presents a simplified block diagram of the power controller. The inputs to the proposed cloud controller are the battery SOC and the torque conversion factor $p$, which is the ratio of the difference $\Delta T$ between the vehicle torque requirement $T_{\text {req }}$ and the ICE optimal torque to the ICE optimal torque $T_{\text {ICE_opt }}$. The output is defined as the normalized ratio of ICE torque command to ICE optimal torque, denoted by $K$. Once $K$ is determined, the ICE torque command ( $\left.T_{\text {ICE }}\right)$ can be made as $K$ multiplied by ICE optimal torque at current condition $\left(T_{\text {ICE }}=K \times T_{\text {ICE_opt }}\right)$, and the EM torque command $\left(T_{\mathrm{EM}}\right)$ is to be the desired vehicle torque minus the ICE torque command $\left(T_{\mathrm{EM}}=T_{\text {req }}-T_{\mathrm{ICE}}\right)$.

4.2. Cloud Controller Implementation. It is actually a kind of mapping that cloud controller realizes the relationship between input and output. The controller is composed of multirule inference part and output data processing section. As shown in Figure 3, the inference part achieves the inference results by $X$-condition cloud and $Y$-condition cloud. To obtain precise value, multirule control can use maximum membership method, reverse cloud method, and weighted average method. Taking into account the realtime characteristic of control, this design uses the weighted average method. Suppose there are $n$ rules in the rule base; when inputs $x_{A}$ and $x_{B}$ activate different rule antecedent $\mathrm{PCG}_{A_{1} B_{1}} \sim \mathrm{PCG}_{A_{n} B_{n}}$, different $\mu_{n i}$ are produced to activate the corresponding rule consequently; then a lot of $\operatorname{drop}_{i}\left(y_{i}, \mu_{n i}\right)$ are created. After dealing with these drops by the weighted average method, output $z$ is obtained. Suppose $p \in A=$ $[-1.5,1.5]$, SOC $\in B=[0,1]$, and $K \in C=[0,1.2]$, where $A$, $B$, and $C$ are all real numbers set. At these three sets, five input cloud models and six output cloud models are separately defined. These models are $A_{1} \sim A_{5}, B_{1} \sim B_{5}$, and $C_{1} \sim C_{5}$, which are expressed as $\left(E_{x}, E_{n}, H_{e}\right)$ by the digital characteristics of normal cloud model. Learning from the idea of fuzzy theory, cloud models are constructed as follows:

$$
\begin{aligned}
& A_{1}=\text { "too low" }=(-1.5,0.35,0.01) ; \\
& A_{2}=\text { "low" }=(-0.5,0.17,0.01) ; \\
& A_{3}=\text { "normal" }=(0,0.17,0.01) ; \\
& A_{4}=\text { "high" }=(0.5,0.17,0.01) ; \\
& A_{5}=\text { "too high" }=(1.5,0.17,0.01) ; \\
& B_{1}=\text { "too low" }=(0,0.1,0.01) ; \\
& B_{2}=\text { "low" }=(0.3,0.07,0.01) ; \\
& B_{3}=\text { "normal" }=(0.6,0.07,0.01) ; \\
& B_{4}=\text { "high" }=(0.75,0.05,0.01) ; \\
& B_{5}=\text { "low" }=(1,0.07,0.01) ;
\end{aligned}
$$




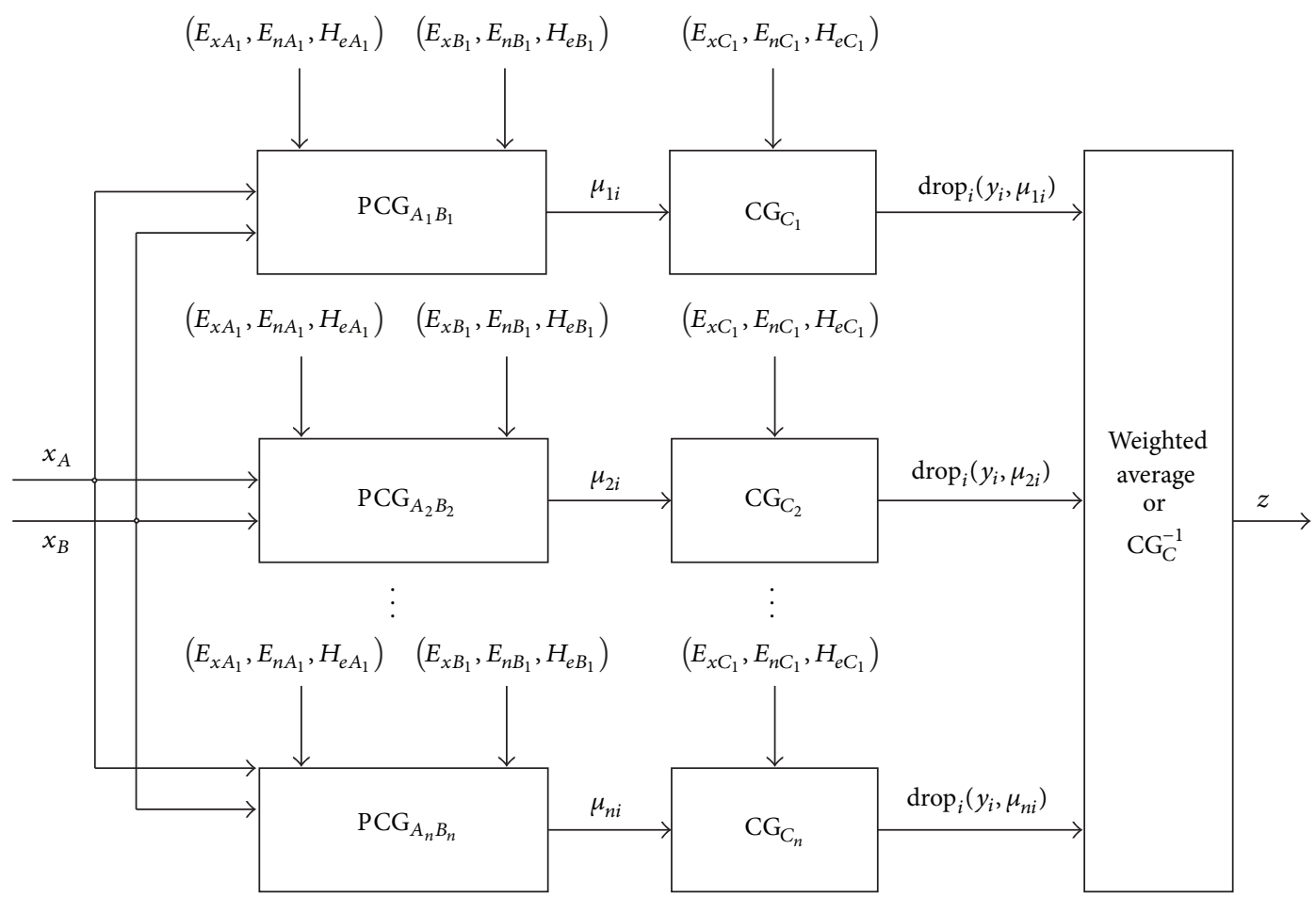

Figure 5: The multirule constructor.

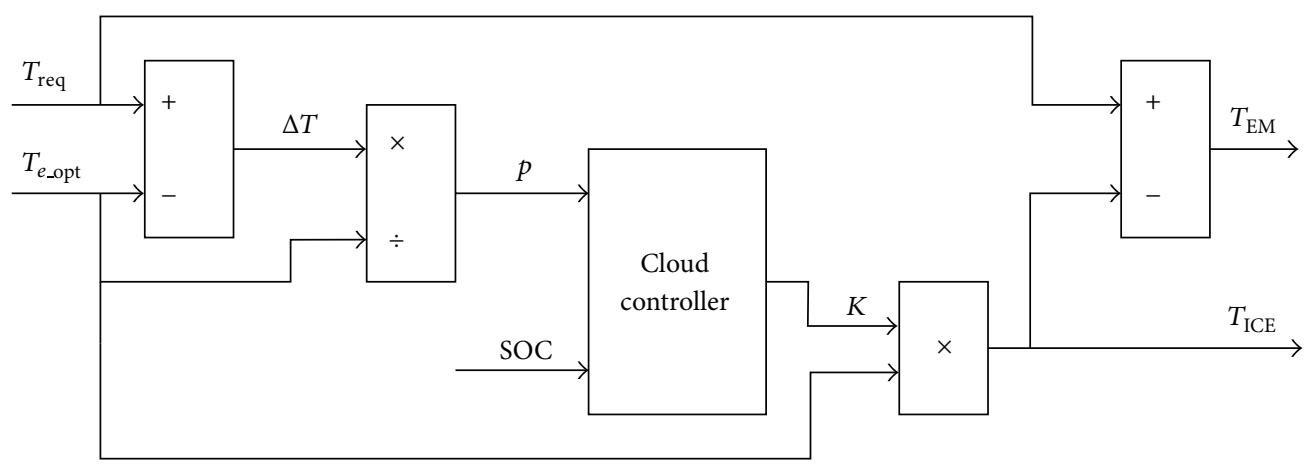

FIGURE 6: Simplified block diagram of the power controller.

$C_{1}=$ "too low" $=(0,0.13,0.01) ;$

$C_{2}=$ "medium low" $=(0.4,0.07,0.01)$

$C_{3}=$ "low" $=(0.6,0.07,0.01)$

$C_{4}=$ "normal" $=(0.8,0.07,0.01) ;$

$C_{5}=$ "high" $=(1.2,0.13,0.01)$.

Similar to the fuzzy controller, the basic idea of a cloud controller is to formulate human knowledge and reasoning, which can be represented as a collection of if-then rules, in a way tractable for computers. The designed cloud controller has 25 inference rules, which are expressed by matrix as follows:

$$
R=\left\{r_{i j}\right\}=\left[\begin{array}{lllll}
4 & 5 & 5 & 5 & 5 \\
4 & 4 & 4 & 4 & 5 \\
3 & 3 & 3 & 4 & 5 \\
2 & 2 & 2 & 3 & 4 \\
1 & 1 & 2 & 2 & 3
\end{array}\right]
$$

where $r_{i j}$ denotes the inference rule "if $A_{i}$ and $B_{j}$, then $C_{k}$, $i=j=1,2, \ldots, 5, k=1,2, \ldots, 6$." For example, $r_{23}$ express the rule "if $A_{2}$ and $B_{3}$, then $C_{4}$."

The above-mentioned cloud model of inputs, outputs, and rules was determined by simulation to increase the system efficiency and to maintain the battery SOC. Using these models and rules, reasoning process from known conditions 


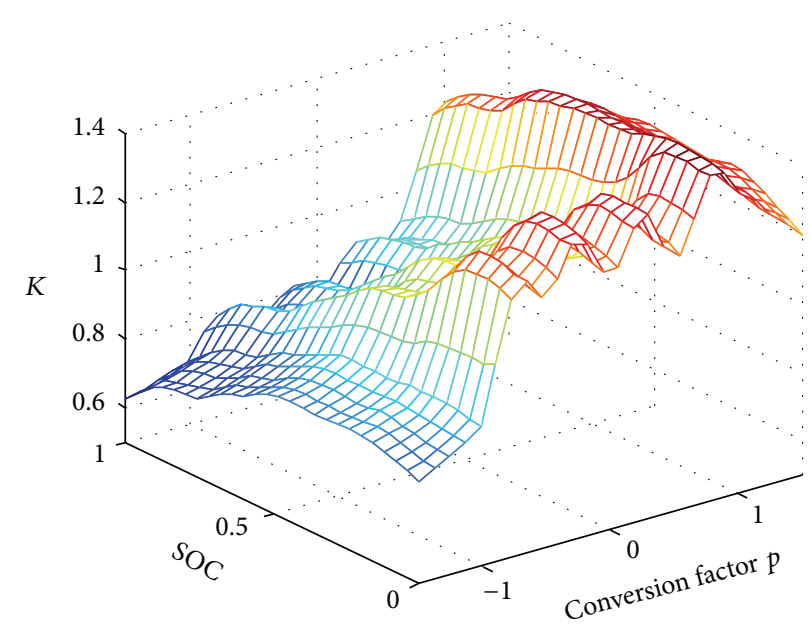

FIGURE 7: Output result of cloud controller.
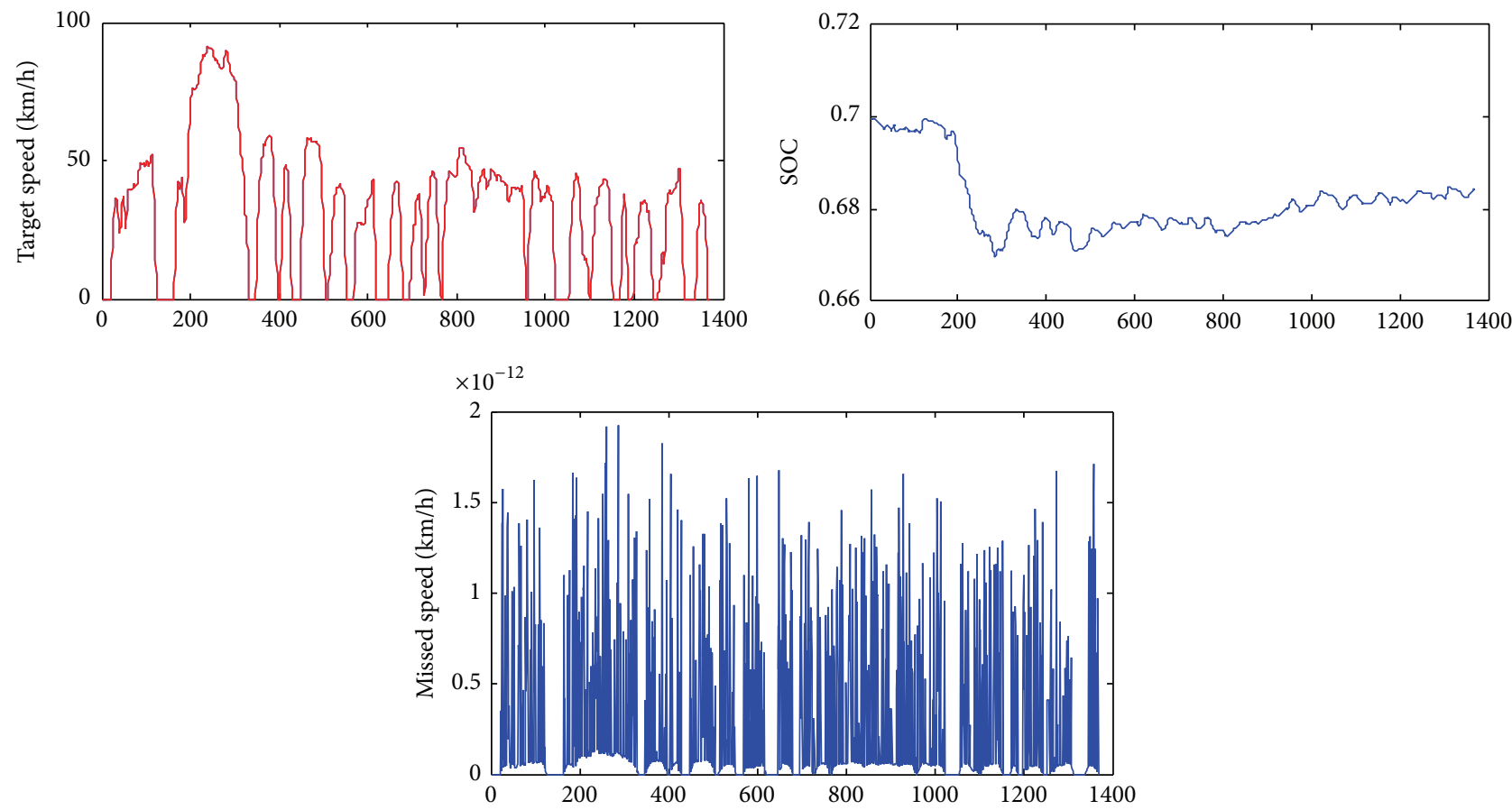

FIGURE 8: Missed speed and SOC history over UDDS driving cycle.

to quantitative output is implemented. Figure 7 shows the result of cloud controller.

\section{Simulation Results}

The advanced vehicle simulator, ADVISOR [24], was used for simulation studies in this work. ADVISOR employs a combined forward/backward facing approach for the vehicle performance simulation. The strategy is tested on four standard driving cycles: the new European driving cycle (NEDC), the urban dynamometer driving schedule (UDDS), the federal test procedure (FTP), and the highway fuel economy test (HWFET). These speed profiles, representing urban and highway scenarios, are widely used in the literature to evaluate the performance of the proposed EMS [25].

As mentioned before, the bottom line for the control strategy is that the vehicle must follow the driver's request and the battery SOC is kept in a certain range. These constraints are adequately satisfied by the proposed EMS; the difference between required and achieved speed (missed speed) as well as the history of SOC over UDDS cycle is shown in Figure 8. It is clear that there is an excellent agreement between the achieved speed and target speed and the charge sustaining requirement is satisfied.

To investigate the effectiveness of the proposed strategy and study the impact of the driving cycle on the cloud control 
TABLE 1: Comparison of fuel consumption and emissions for four cycles.

\begin{tabular}{lccccccc}
\hline & \multicolumn{2}{c}{ UDDS } & \multicolumn{2}{c}{ FTP } & \multicolumn{2}{c}{ HWFET } & \multicolumn{2}{c}{ NEDC } \\
& FLC & Cloud & FLC & Cloud & FLC & Cloud & FLC \\
\hline FC $(1 / 100 \mathrm{~km})$ & 6.0 & 5.5 & 5.8 & 5.3 & 4.3 & 3.7 & 5.7 \\
$\mathrm{HC}(\mathrm{g} / \mathrm{km})$ & 0.368 & 0.34 & 0.283 & 0.262 & 0.263 & 0.23 & 0.379 \\
$\mathrm{CO}(\mathrm{g} / \mathrm{km})$ & 1.044 & 1.096 & 0.79 & 0.828 & 0.829 & 0.87 & 1.049 \\
$\mathrm{NO}_{x}(\mathrm{~g} / \mathrm{km})$ & 0.319 & 0.278 & 0.259 & 0.226 & 0.252 & 0.206 & 0.359 \\
\hline
\end{tabular}

TABLE 2: Comparison of component losses for two controllers for four driving cycles.

\begin{tabular}{ccccc}
\hline & ICE losses $(\mathrm{kWh})$ & Electric motor $(\mathrm{kWh})$ & Transmission losses $(\mathrm{kWh})$ & Battery losses $(\mathrm{kWh})$ \\
\hline UDDS & & & & 0.089 \\
FLC & 4.68 & 0.034 & 0.089 & 0.087 \\
Cloud & 4.37 & 0.050 & & 0.13 \\
FTP & & & 0.13 & 0.13 \\
FLC & 6.56 & 0.053 & & 0.13 \\
Cloud & 6.14 & 0.076 & 0.13 & 0.054 \\
HWFET & 4.40 & & 0.13 & 0.054 \\
FLC & 3.93 & 0.039 & & 0.073 \\
Cloud & & 0.065 & 0.097 & 0.067 \\
NEDC & 4.06 & & 0.096 & \\
FLC & 3.79 & 0.031 & & \\
Cloud & & 0.05 & & \\
\hline
\end{tabular}

strategy, the simulation is also performed over FTP, HWFET, and NEDC driving cycles. In addition, for comparing the results, the same simulations have been done with the default FLC based on efficiency mode in ADVISOR, which is similar to the proposed EMS with optimizing ICE operation. Table 1 shows the fuel consumption and emissions of both controllers for four driving cycles. To compare with the FLC, the fuel consumption of powertrain with cloud controller is fewer for all four cycles. To the HC and NOx emissions, the proposed controller is better than the FLC. However, the CO emission is worse for all four cycles.

Table 2 presents the component losses for four cycles. The losses of transmission and battery are almost the same for four cycles with two mentioned controllers. The EM loss for four cycles with cloud controller is higher than the cycles with FLC. However, the overall efficiency of the cloud controller is better than of the FLC since the ICE loss for the cycles with cloud controller is far less than with the FLC.

\section{Conclusions}

A cloud model-based energy management strategy for parallel hybrid vehicles (PHVs) is presented. The objective of the proposed EMS is to minimize the fuel consumption and maintain the battery SOC within its operational range while satisfying the driver requirements. The EMS is evaluated in a simulation environment using four standard driving cycles. The results show that the proposed strategy provides an improvement in fuel economy and has stronger robustness.

\section{Conflict of Interests}

The authors declare that there is no conflict of interests regarding the publication of this paper.

\section{Acknowledgments}

The support of the National Natural Science Foundation of China (Grant no. 51405367) and the Key Laboratory of Road Construction Technology and Equipment of China (Grant no. 310850130169 ) is much appreciated.

\section{References}

[1] J. Seiler and D. Schröder, "Hybrid vehicle operating strategies," in Proceeding of the 15th Electric Vehicle Symposium (EVS '98), Bruxelles, France, 1998.

[2] B. M. Baumann, G. Washington, B. C. Glenn, and G. Rizzoni, "Mechatronic design and control of hybrid electric vehicles," IEEE/ASME Transactions on Mechatronics, vol. 5, no. 1, pp. 5872, 2000.

[3] N. J. Schouten, M. A. Salman, and N. A. Kheir, "Fuzzy logic control for parallel hybrid vehicles," IEEE Transactions on Control Systems Technology, vol. 10, no. 3, pp. 460-468, 2002.

[4] F. G. Harmon, A. A. Frank, and S. S. Joshi, "The control of a parallel hybrid-electric propulsion system for a small unmanned aerial vehicle using a CMAC neural network," Neural Networks, vol. 18, no. 5-6, pp. 772-780, 2005.

[5] J. S. Won and R. Langari, "Intelligent energy management agent for a parallel hybrid vehicle-part II: torque distribution, charge 
sustenance strategies, and performance results," IEEE Transactions on Vehicular Technology, vol. 54, no. 3, pp. 935-953, 2005.

[6] M. Montazeri-Gh, A. Poursamad, and B. Ghalichi, "Application of genetic algorithm for optimization of control strategy in parallel hybrid electric vehicles," Journal of the Franklin Institute, vol. 343, no. 4-5, pp. 420-435, 2006.

[7] L. V. Pérez, G. R. Bossio, D. Moitre, and G. O. García, "Optimization of power management in an hybrid electric vehicle using dynamic programming," Mathematics and Computers in Simulation, vol. 73, no. 1-4, pp. 244-254, 2006.

[8] A. Sciarretta, M. Back, and L. Guzzella, "Optimal control of parallel hybrid electric vehicles," IEEE Transactions on Control Systems Technology, vol. 12, no. 3, pp. 352-363, 2004.

[9] O. Barbarisi, E. R. Westervelt, F. Vasca, and G. Rizzoni, "Power management decoupling control for a hybrid electric vehicle," in Proceedings of the 44th IEEE Conference on Decision and Control, and the European Control Conference (CDC-ECC '05), pp. 2012-2017, December 2005.

[10] P. Pisu and G. Rizzoni, " $H_{\infty}$ control for hybrid electric vehicles," in Proceedings of the 43rd IEEE Conference on Decision and Control (CDC '04), vol. 4, pp. 3497-3502, December 2004.

[11] L. De-yi, M. Hai-jun, and S. Xue-mei, "Membership clouds and membership cloud generators," Journal of Computer Research and Development, vol. 32, no. 6, pp. 15-20, 1995.

[12] D. Y. Li, "The cloud control method and balancing patterns of triple link inverted pendulum systems," Engineering Sciences, vol. 1, no. 2, pp. 41-46, 1999.

[13] L. Zhang, F. Sun, and Z. Sun, "Cloud model-based controller design for flexible-link manipulators," in Proceedings of the IEEE Conference on Robotics, Automation and Mechatronics, pp. 1-5, IEEE, Bangkok, Thailand, June 2006.

[14] D. Kaichang, L. Deyi, and L. Deren, "Knowledge representation and discovery in spatial databases based on cloud theory," International Archives of Photogrammetry and Remote Sensing, vol. 32, pp. 544-551, 1998.

[15] S. Wang, D. Li, W. Shi, D. Li, and X. Wang, "Cloud model-based spatial data mining," Geographic Information Sciences, vol. 9, no. 1-2, pp. 60-70, 2003.

[16] F. Wang, Y. Li, and X. Li, "A new performance evaluation method for automatic target recognition based on forward cloud," in AsiaSim 2007, vol. 5 of Communications in Computer and Information Science, pp. 337-345, Springer, Berlin, Germany, 2007.

[17] R. He, J. Niu, M. Yuan, and J. Hu, "A novel cloud-based trust model for pervasive computing," in Proceedings of the 4th International Conference on Computer and Information Technology, pp. 693-700, IEEE, September 2004.

[18] C.-H. Dai, Y.-F. Zhu, and W.-R. Chen, "Adaptive genetic algorithm based on cloud theory," Kongzhi Lilun Yu Yinyong, vol. 23, no. 4, pp. 646-650, 2007.

[19] P. Lv, L. Yuan, and J. Zhang, "Cloud theory-based simulated annealing algorithm and application," Engineering Applications of Artificial Intelligence, vol. 22, no. 4-5, pp. 742-749, 2009.

[20] X. Wu, B. Cao, J. Wen, and Z. Wang, "Application of particle swarm optimization for component sizes in parallel hybrid electric vehicles," in Proceedings of the IEEE Congress on Evolutionary Computation (CEC '08), pp. 2874-2878, IEEE, 2008.

[21] D. Li, J. Han, X. Shi, and M. C. Chan, "Knowledge representation and discovery based on linguistic atoms," Knowledge-Based Systems, vol. 10, no. 7, pp. 431-440, 1998.
[22] C. Yang and D. Li, "Planar model and its application in prediction," Chinese Journal of Computers, vol. 21, no. 11, pp. 961-969, 1998.

[23] A. Poursamad and M. Montazeri, "Design of genetic-fuzzy control strategy for parallel hybrid electric vehicles," Control Engineering Practice, vol. 16, no. 7, pp. 861-873, 2008.

[24] D. Feroldi, M. Serra, and J. Riera, "Energy management strategies based on efficiency map for fuel cell hybrid vehicles," Journal of Power Sources, vol. 190, no. 2, pp. 387-401, 2009.

[25] T. Markel, A. Brooker, T. Hendricks et al., "ADVISOR: a systems analysis tool for advanced vehicle modeling," Journal of Power Sources, vol. 110, no. 2, pp. 255-266, 2002. 

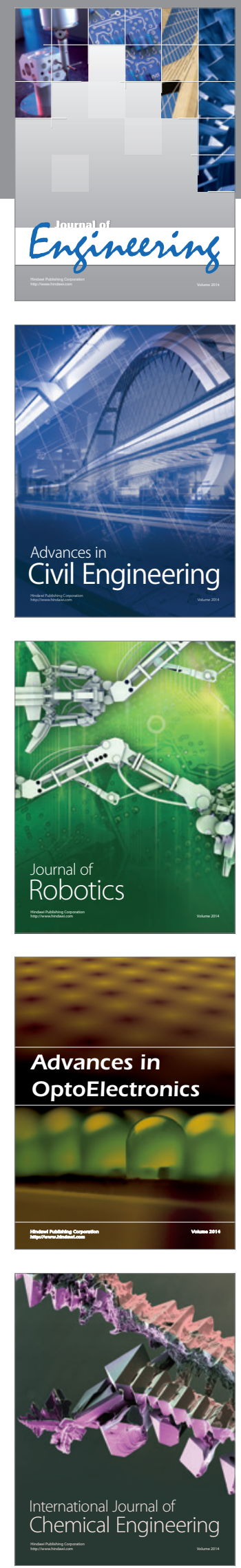

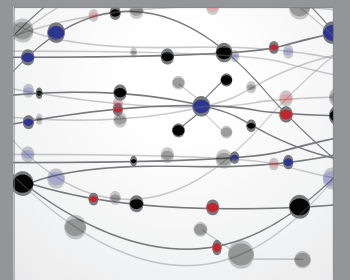

The Scientific World Journal
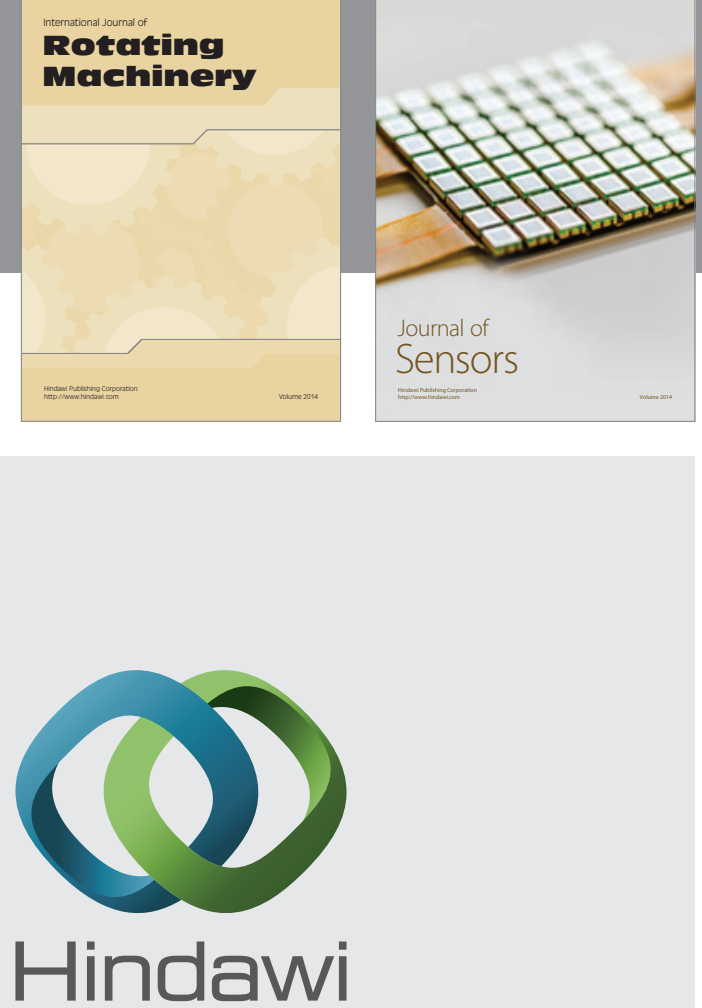

Submit your manuscripts at http://www.hindawi.com
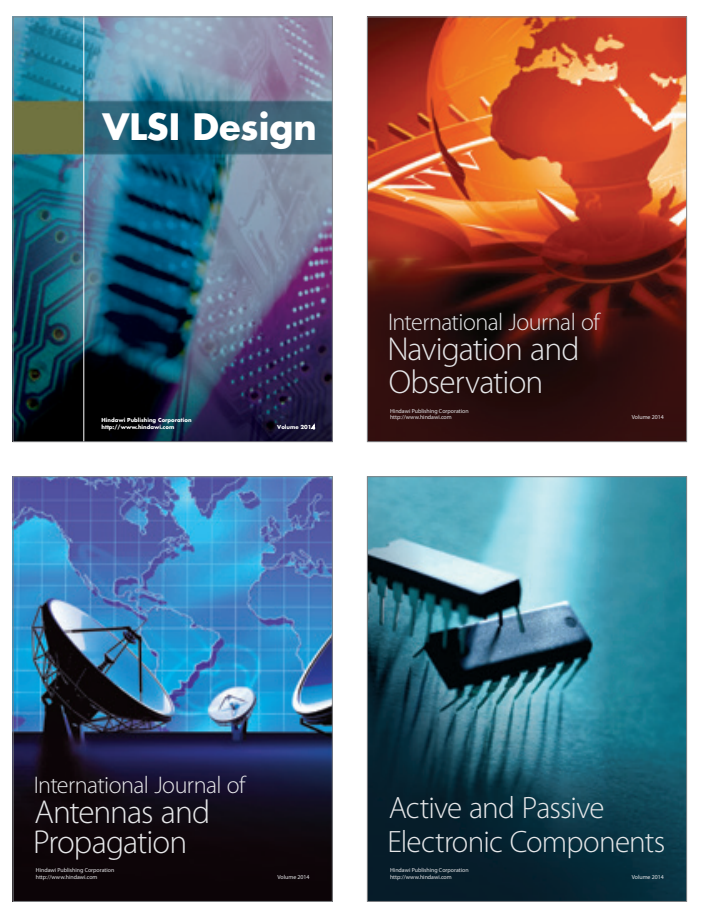
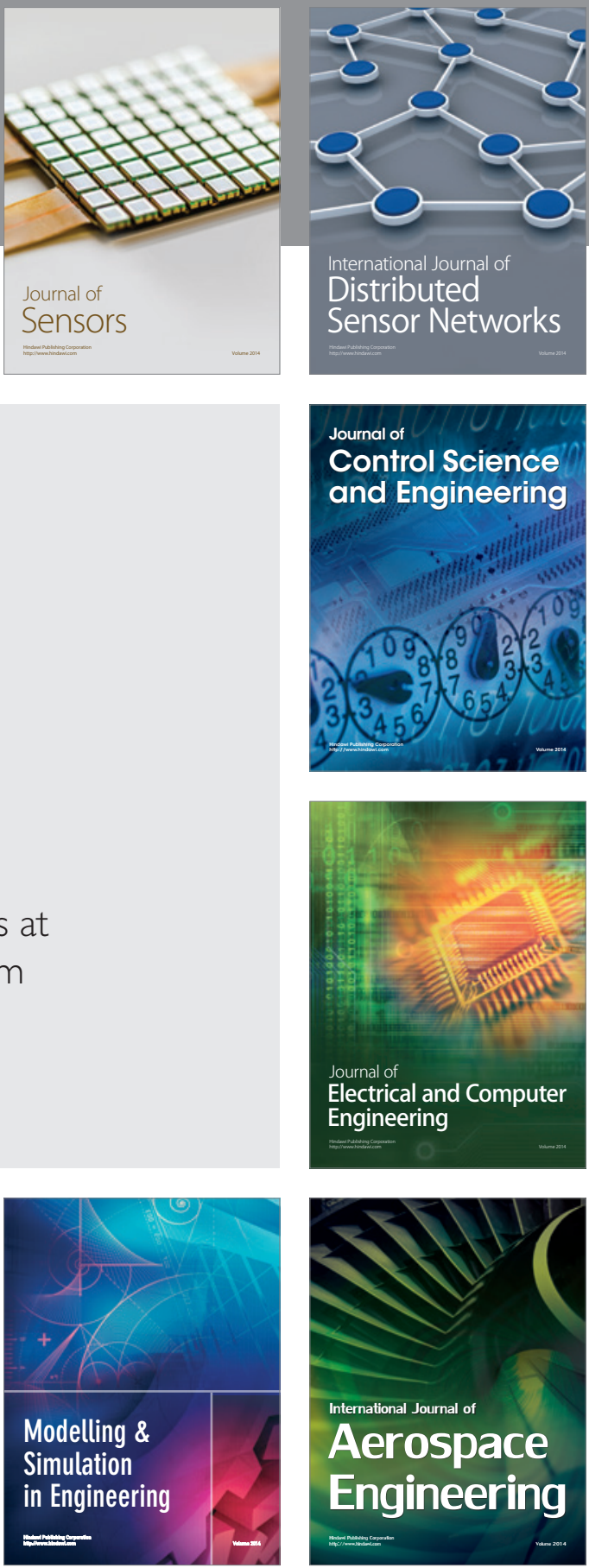

Journal of

Control Science

and Engineering
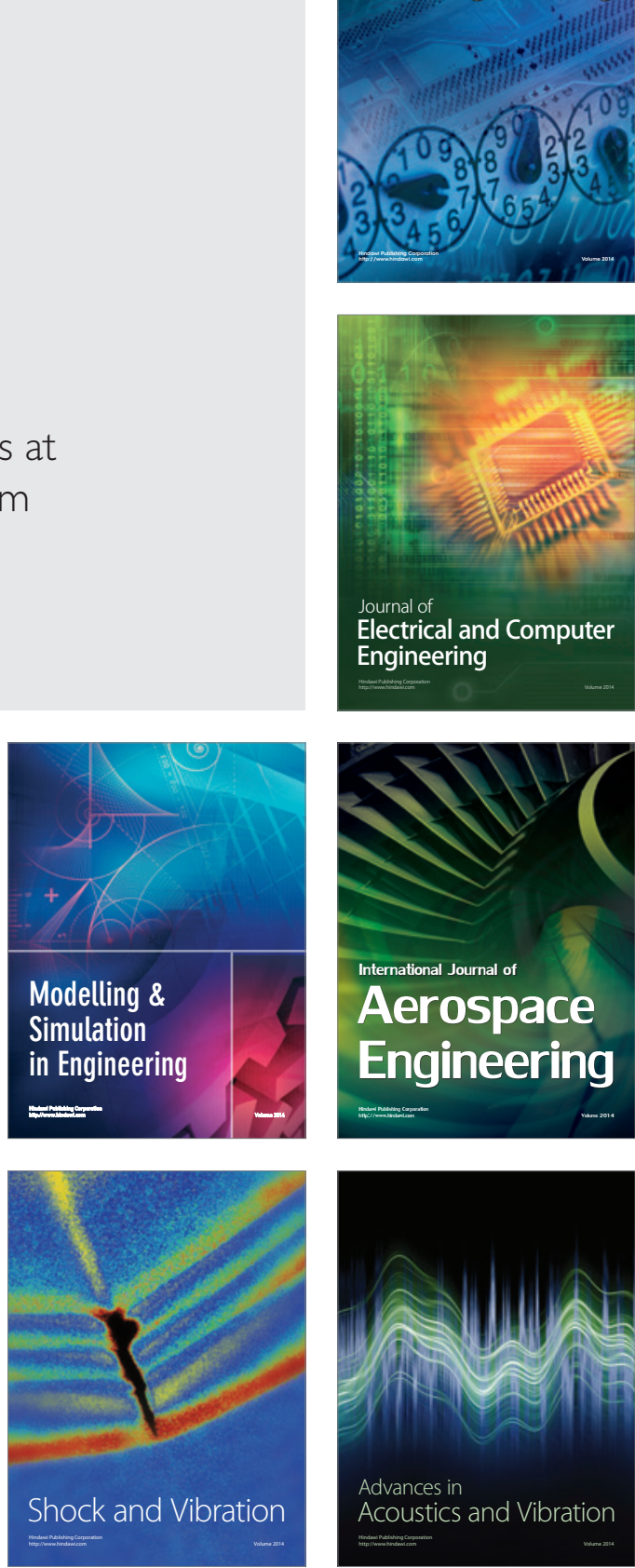